\title{
Erwiderung auf Herrn Hoorweg's Abhandlung betr. „Ueber die Nervenerregung durch Condensator- entladungen."
}

\author{
Von
}

\section{N. Cybulski und J. Zanietowski.}

Im zehnten Hefte dieses Archivs (Bd. 57, S. 427-437) finden wir eine kurze Abhandlung von H. J. L. Hoorweg; "Ueber die Nervenerregung durch Condensatorentladungen", in welcher der Verfasser bei Besprechung der von uns in demselben Archiv (Bd. 56 S. 45-148) beschriebenen Reizungsmethode die Hoffnung hegt, uns iberzengen zu können, dass, obwohl unsere Versuchsmethode "einwurfsfrei und mit Sorgfalt durchgeführt" ist, doch die Resultate derselben wegen mangelhafter Condensatoren, allen Werth entbehren" (S. 431).

Wir müssen jedoch vor allem die Aufmerksamkeit des Herrn Hoorw eg darauf lenken, dass der Zweck seiner Experimente und derjenige der unsrigen ganz verschieden sind. Während nämlich Herr Hoorweg dahin strebte, eine mathematische Formel der Erregung auszusuchen, bemühten wir uns, die Lücke, welche in dem Mangel einer genauen Reizmethode besteht, anszufiullen, ohne jedoch zu glauben, so wie Herr Hoorweg es ausdrücklich wiederholt (S. 427), „die Energie als Maass der elektrischen Erregung gefunden zu haben."

Da nun von allen Arten der bisher angewandten elektrischen Reizungen nur die Reizung durch Condensatorentladungen am besten eine exacte Bestimmung der physikalischen Bedingungen ermöglichte, haben wir genau die Wirkung der Condensatorentladungen auf Nerven und Muskeln studirt, und uns nach langen Proben eine möglichst praktische und doch gena ue Versuchseinrichtung zu konstruiren bemüht, die dem oben erwähnten Zwecke entsprechen wirde. Dieses doppelte Ziel der praktischen Brauchbarkeit und der wissenschaftlichen Genauigkeit hatten wir zuerst im Auge, sowohl in der Auswabl der einzelnen Bestandtheile, wie 
auch in der Einrichtung der ganzen Reizungsmethode. Erst als dieselbe uns, ,ein w u rfsfrei" (so wie sie Herr Hoorweg selbst nennt, S. 431) zu sein schien, haben wir die Abhängigkeit des physiologischen Reizeffektes ron dem Potential, der Elektricitätsquantität und der Energie gesucht. Da nun in diesen Versuchen eine gewisse Relativität zwischen der Energie des Reizes und der Zeit, in welcher die Ladung den Nerv durchströmt, einerseits, und dem physiologischen Effecte andererseits sichtbar war; da wir zweitens in zablreichen Versuchen bei verschiedenen Quantitäten und derselben Energie dieselbe Zuckung erhielten, haben wir die Hypothese aufgestellt, dass die Erregung von der Energie und der Zeit der Entladung abhängig ist, und aus diesem Grunde in einigen Versuchsreihen den Reiz in Ergen ausgedrückt. Daneben bemerkten wir es ausdrücklich (S. 97), dass, wenn auch die 'Ab. hängigkeit von der Erregungsenergic, die wir in unseren Experimenten fanden, durch weitere Forschungen nicht bestätigt worden wäre, die Thatsache, dass sich die elektrische Wirkung auf den Nerv in Einheiten der Energie ausdrücken lässt," uns dazu führen musste, den Reiz in diesen Einheiten auszudrücken, da auf diese Weise wir die innere und äussere Energie der Zuckung mit der Energie des Reizes vergleichen könnten. Von diesem rein $\mathrm{prakt}$ is c he n Standpunkte ansgehend, haben wir den Reiz in Einheiten der Energie ausgedrickt, ohne jedoch es zu vermeiden, die physiologisehen Effecte auch mit dem Zuwachs der Polspannung, Quantität u. s. w. zu vergleichen.

Kurz gesagt, war also unsere Aufgabe ganz praktisch, während Herr $\mathrm{H}$ o o rw eg in seiner Arbeit dabin gestrebt hat, die Art und Weise der Wirkung der Elektricität zu erklären, und auf Grund seiner Experimente eine empirische Formel anfzustellen, welche eine ,mathematische Vorstellung des allgemeinenGrundgesetzes derelektrischen Nervenerregang" sein soll (S. 436). Endlich verwirft er (ibid. S. 433) „alle diejenigen Erregungsgesetze, welehe für Condensatorentladungenzueiner anderenalszu dieser Formel leiten".

Was den Hauptrorwurf anbelangt, als ob unsere Condensatoren (von S i e m e n s) ibrem Ziele gar nicht entsprechen sollten, muissen wir ihn als zu umfangreich ansehen. Herr $\mathrm{H}$ o $0 \mathrm{rw}$ eg sagt nämlich auf S. $431:$,Um so mehr ist es zu bedanern, 
dass allein wegen der mangelhaften Isolation der Condensatoren die Resultate der Herren C. und $Z$ allen Werth en tbehren". Um uns za überzeugen, was für einen Einfluss diese mangelhafte Isolation hat, wird es genügen, unsere Experimente mit denjenigen des Herrn $H$. zu vergleichen.

Nehmen wir z. B. einige Versuche in unserer Abhandlung (S. $81-83$ ) und in Herrn Hoorw eg's Antwort S. 432). Bei der Anwendung von Condensatoren von $2000.10^{-11}$ und $500.10^{-11}$ (die wir deswegen wählen, da sich dieselben Capacitäten anch in unseren Versuchen befinden) erhält Herr $\mathrm{H}$ o $0 \mathrm{r}^{\mathrm{r}} \mathrm{w}$ e folgende (znm Hervorrufen der minimalen Zuckung nöthige) Quantitäten:

\begin{tabular}{|c|c|}
\hline Condens. $2000.10^{-11}$ & Condens, 500.10-11 \\
\hline 490.10-11 Coulombs & 309.10-11 Coulombs \\
\hline $260.10^{-11}$ & $156.10^{-11}$ \\
\hline
\end{tabular}

Wir erhielten die minimale Zuckung in ähnlichen Versuchen I., II. and III. Versuch S. $81-83$ ) bei folgenden Quantitäten:

\begin{tabular}{|c|c|}
\hline Condens. $2000.10-11$ & Condens. $500.10-11$ \\
\hline 372.10-11 Coulombs & 234.10-11 Coulombs \\
\hline $416.10-11 \mathrm{C}$ & $252.10-11 \mathrm{C}$. \\
\hline $321.10-11 \mathrm{C}$ & $164.10^{-11} \mathrm{C}$ \\
\hline
\end{tabular}

Die Schwankungen sind also bei Herrn Hoorweg 230 und 143, bei uns 95 und 88. Daraus ist es leicht ersichtlich, dass beinahe alle unsere Zahlen in den Grenzen derjenigen fallen, die Herr $\mathrm{H}$. angibt. Diese Uebereinstimmung zeigt uns, dass, wenn man einen Vorwurf unseren Zahlen machen will, derselbe zuerst Herrn $\mathrm{Ho}$ orw eg's Zahlen treffen muss, welcher die möglichst besten Condensatoren gebrauchte, oder dass unsere Condensatoren ebenso gut sind, wie diejenigen des Herrn Hoorweg. Warum also unsere Experimente nicht wenigstens diesen „Werth haben sollen, wie Herm Hoorwe g's Versuche, ist schwer zu verstehen. Die Zahlen stimmen nur dort nicht überein, wo wir die kleinsten Conden- 
satoren benutzten (z. B. 55.10-11 F.), von denen wir lange gewusst haben, dass man mit ihnen sehr sorgfältig experimentiren muss. Deswegen gebranchten wir entweder Mica-Condensatoren oder anch Paraffin-Condensatoren, in welchen die isolirende Schichte jedoch nicht aus einem, sondern aus mehreren Paraffinpapierblättern bestand. Da nach diesem allgemeinen Vorwurfe uns Herr Hoorwe $g$ keine Experimente mit solchen Mica-Condensatoren vorbringt, wie unsere kleinsten Paraffincondensatoren, können wir uns nicht so leicht davon ubberzeugen, dass diese Condensatoren wirklich so "lech" sind und "einen messbaren Widerstand dem Strom bieten" (S. 431), wobei wir jedoch nicht leugnen wollen, dass ein minimales Electricitätsquantum wegen Bestäubung der Contacte oder irgend einer kleinen Ungenauigkeit der Verbindung verloren gehen könnte. Mit diesem Punkte werden wir uns noch auf einer anderen Stelle beschäftigen; jedoch glauben wir, dass, wenn auch Herr Hoorweg Recht hätte, dass unsere Zahlen wegen Verlust der Electrizität nur in e inige n Fällen zu gross wären, diese Thatsache doch den Werth seiner Formel nicht retten wird.

Einige, mit einem Luftcondensator (wo die Isolirung also ganz sicher war) durchgeführten Experimente haben uns überzengt, was schon von unseren früheren Versuchen bestätigt wurde (S. 104), dass es ein anderes Verhältniss zwischen dem Potential und dem eingeschalteten Widerstande gibt, als es Herr Hoorweg denkt; seine Formel lautet nämlich

$$
\boldsymbol{t}=a R+\frac{b}{C}
$$

während der Versuch uns zeigt, dass, je grösser der eingeschaltete Widerstand des Nerven ist, die Polspannung und die Quantität desto kleiner sein müssen, um eine minimale Zuckung hervorzu= rufen. Als Beispiel fiihren wir hier nur zwei Versuche an, in welchen wir, um immer denselben Contact mit dem Nerven zu erhalten, den Nerven so auf die unpolarisirbaren Elektroden hinlegten, dass man dureh einfaches Auseinandersehieben der Electroden einmal $2 \mathrm{~mm}$ und wiederum $30 \mathrm{~mm}$ desselben Nerven reizen konnte, ohne dessen Lage auf den Electroden za ändern. 


\begin{tabular}{|c|c|c|c|}
\hline \multicolumn{4}{|c|}{ Condens. $109.10^{-11} \mathrm{~F}$. } \\
\hline $\begin{array}{c}\text { Widerstand des } \\
\text { Nerven }\end{array}$ & Volts & Coulombs & Ergs \\
\hline 62300 Ohms & 1,455 & $162,05,10-11$ & 0,0106 \\
\hline $221600 \Omega$ & 0,945 & $102,4.10-11$ & 0,004 \\
\hline
\end{tabular}

Condens. 188.10-11 $\mathrm{F}$

\begin{tabular}{r|r|r|r}
$23300 \Omega$ & 1,58 & $297,04 \cdot 10-11$ & 0,023 \\
$191300 \Omega$ & 1,09 & $204,92.10-11 !$ & 0,011
\end{tabular}

Dasselbe war schon aus den Tabellen auf S. 103, 104 unserer Abhandlung ersichtlich, wo zwar die Coulombs nicht angegeben sind, aber wo die Reochordlänge desto mehr wächst, je kleiner der Abstand der Elektroden, und was dessen Folge ist, der Widerstand der Nerven ist. Wie kann man nun die Formel

$$
P=a R+\frac{b}{C}
$$

erklären, wo $P$ mit dem Zuwachs von $R$ wachsen soll, wenn $a, b$ und $C$ constant bleiben.

'Wweitens haben unsere Experimente, in welchen wir den Zawachs der Muskelznckung mit dem Zuwachs der Elektrizitätsmenge za vergleichen versucht baben $(S .114,116)$, es sehon genau gezeigt, dass, wenn die Elektrizitätsmenge um ein gewisses Quantum wächst, der Zuwachs der Zuckungshöhe und der Arbeit nicht gleichmässig ist; beide wachsen nämlich anfangs langsam, dann schneller, und nachdem sie eine gewisse Höbe erreicht haben bleiben sie trotz des fortdauernden weiteren Zuwachses des Reizes auf derselben stehen. Diese Thatsache, welche doch uberhaupt nicht gelengnet werden kann, dass es ein Maximum der Erregung gibt, welches man trotz Verstärkung des Reizes nicht überschreiten kann, steht doch keineswegs in Uebereinstimmung mit der von Herrn Hoorweg citirten ${ }^{1}$ ) Formel

$$
y=\frac{\alpha_{0} P C}{\beta R C+1}
$$

woraus es doch klar einleuchtet, dass die Totalerregung $\mathbf{y}$ mit der Quantität $P C$ proportional wachsen muss. Zwar sagt Herr

1) "Ueber eine neue Methode der elektrodiagnost. Untersuchung." Deutsch. Arch, für klin. Med, Bd. 51. S. 208. 
Hoorweg in der Fortsetzung der erwähnten Abhandlung (Deutsch. Arch. für klin. med., Bd. 52, S. 551). „Es scheint, dass meine Abhandlung in Pfl ïger's Archiv hier und dort an Deutlichkeit $\mathrm{z} u$ wünschen übrig gelassen hat, denn viele hegen die Meinung; ich habe die Erregung einfach der Quantität Elektrizität proportional gefunden, während ich doch in Wirklichkeit zu einem ganz anderen Resultate gelangte."

Diese Bebauptung ist wahr, was die Deutlichkeit anbetrifft, weil wir uns anders die erwähnte Formel gar nicht erläutern können.

Dass in der Arbeit des Herrn H. sich einige andere Undeutlichkeiten befinden, lässt sich nicht leugnen, wie zum Beispiel: Auf S. 554 sagt Herr H. selbst, dass die Einheit der Erregung diejenige, Erregung ist, welche ,die minimale $\mathrm{Zuck}$ ung anszulösen im Stande ist"; auf S. 553 finden wir aber, dass „die Untersuchungen... über die Grösse der Zuckungen bei verschiedenen Stromstärken nicht mehr die Erregung, sondern die Zuckung zum Zweck baben". Wie man das verstehen, wie man überhaupt die Verănderungen der Erregung untersuchen soll, ohne die Zackung zu beobachten, ist uns unbegreiflich. Dazu beschreibt Herr $H$. so wenige Experimente und so wenig genau, dass wir nicht wissen, wie z. B. die als Erregungseinheiten aufgefassten Zuckungen beobachtet waren; ob er z. B. das Minimum unter dem Mikroskop, oder mit blossem Auge, oder vermittelst eines Myographions beobachtet hat, bleibt uns unbekannt, während doch die Art der Bestimmung des Minimum nicht gleichgültig sein kann.

Die oben erwähnten Experimente zum Studium des Verhältnisses zwischen Potential und Widerstand einerseits, zwischen Zackung und Elektrizitätsquantität andererseits, scheinen uns genügend zu beweisen, dass Herm Hoorweg's Formel nicht so leicht als ,allgemeines Grundgesetz der elektrischen Nervenerregung" angenommen werden kann, wenigstens was die Nervenerregung vermittelst Condensatorentladungen betrifft. 